\section{RADIOTERAPIA PREOPERATORIA EN EL ADENOCARCINOMA LOCALIZADO DE RECTO Y METODOLOGÍA DE LOS ESTUDIOS CLÍNICOS}

\section{Estimado director:}

Hemos leído con interés un artículo original sobre la radioterapia preoperatoria en el cáncer de recto, publicado recientemente en esta revista ${ }^{1}$, que pensamos merece ciertas reflexiones. Lo primero que Ilama la atención en él es el diseño del estudio: la frase «el propósito fue realizar un estudio retrospectivo» traduce un error (conceptual o lingüístico), ya que el objetivo del trabajo no puede ser él en sí mismo. Por otra parte, si es un estudio retrospectivo, no se trata evidentemente de un estudio comparativo aleatorizado; se habla de «distribución aleatoria» lo que, en el contexto citado, parece implicar que cada paciente se trató (con o sin radioterapia preoperatoria) a criterio del cirujano y, por tanto, sin un protocolo terapéutico establecido. En otro punto los autores refieren que los pacientes estaban «repartidos de forma homogénea con respecto a edad y estadios». ¿Significa esto que estamos ante un estudio de casos apareados? Si ello fuera así, el resultado habría sido insatisfactorio, ya que la distribución por sexos (63,5\% de varones en un grupo y $44,4 \%$ en el otro) y la mediana de distancia del tumor al margen anal (8 frente a $14 \mathrm{~cm}$ ) fueron Ilamativamente desiguales. En cualquiera de los casos, no presentan los autores ningún análisis estadístico de factores pronóstico que garantice la comparabilidad de los 2 grupos.

Un segundo aspecto cuestionable es la ausencia de quimioterapia adyuvante en ambas ramas de tratamiento y la omisión de radioterapia postoperatoria en el brazo de cirugía, sin que se manifieste justificación alguna al respecto. Los pacientes se trataron entre 1990 y 1996, pero ya desde 1990 existe un consenso internacional sobre la necesidad de administrar un tratamiento combinado (quimioterapia y radioterapia) adyuvante (preoperatorio o postoperatorio) en el cáncer de recto ${ }^{2}$. En el apartado de smaterial y métodos» sólo se describe la técnica de irradiación, obviándose el protocolo quirúrgico, el grado de especialización de los cirujanos y la realización de escisión mesorrectal total (factores pronóstico de primer orden). Desconocemos cómo se realizó la estadificación preoperatoria de los pacientes, ya que no se menciona la ecografía endorrectal ni la resonancia magnética, únicas técnicas aceptadas hoy día para esta localiza- ción. Esto nos impide conocer realmente el tumoradenopatía-metástasis (TNM) inicial de los pacientes tratados y, por tanto, la comparación de los estadios de Dukes postoperatorios es imposible de interpretar. Se menciona un índice de recidivas locales del 58\% en el grupo de cirugía (cifra preocupante) y no se explica por qué un $18 \%$ de los enfermos tratados con radioterapia neoadyuvante no llegó a operarse.

Con estos defectos metodológicos nada puede concluirse. El estudio no refrenda ni refuta los beneficios de la radioterapia preoperatoria. Es curioso que los autores afirmen que «todos los ensayos al eatorizados (publicados en revistas internacionales) presentan defectos de diseño». La discusión del trabajo es intrascendente y poco actualizada; las citas bibliográficas (algo descuidadas, al menos los números 13, 15 y 16 no coinciden con sus referencias) son en general anteriores a 1995 y no hay ninguna posterior a 1999. Creemos, por tanto, que este trabajo no aporta información científica valorable sobre el tema en que versa. Todo ello reconociendo los méritos del tratamiento neoadyuvante del cáncer de recto localizado, opción que por otra parte defendemos desde hace algún tiempo ${ }^{3}$.

J orge Aparicio Urtasun

Servicio de Oncología Médica. Hospital Universitario La Fe. Valencia.

\section{Bibliografía}

1. Fernández Fernández MC, Errazquin Sáenz de Tejada L, J iménez Gamero MD, Cordero Fernández C. Radioterapia preoperatoria frente a cirugía como tratamiento en el carcinoma de recto. Rev Oncología 2001; 3: 22-27.

2. NIH Consensus Conference. Adjuvant therapy for patients with colon and rectal cancer. JAMA 1990; 264: 1.444-1.450.

3. Fernández-Martos C, Aparicio J, Bosch C, et al. Pre-operative therapy with oral uracil and tegafur (UFT) and concomitant irradiation in operable rectal cancer. Preliminary results of a multicenter phase II study. Proc Am Soc Clin Oncol 2001; 20: 590.

Rev Oncología 2001; 3: 328.

Correspondencia: Dr. J . Aparicio Urtasun. Servicio de Oncología Médica.

Hospital Universitario La Fe.

Avda. Campanar, 21

46009 Valencia.

Correo electrónico: japaricio@ene.es 